\title{
Laser shock processing as a method of decreasing fatigue of a die-casting die made of maraging steel
}

\author{
Janez Grum and Martin Zupančič \\ Faculty of Mechanical Engineering \\ University of Ljubljana \\ Aškerčeva 6, 1000 Ljubljana, Slovenia \\ J.L. Ocaña, M. Morales and J.A. Porro \\ Departamento de Física Aplicada a la Ingeniería Industrial \\ and Centro Láser UPM \\ Universidad Politécnica de Madrid \\ Ctra. de Valencia. km. 7.3. 28031 Madrid. Spain
}

\begin{abstract}
Laser Shock Processing (LSP) is a process of laser treatment with a pulsed beam of high-power density. The process generates locally limited mechanical waves that increase the through-depth density of dislocations. This entails a change in mechanical properties, particularly at the workpiece surface.

The treatment with laser-induced shock waves is suitable for structural parts and machine elements subjected to high thermo-dynamical loads. LSP can substantially improve the wear resistance, which is of exceptional significance to die-casting tools made of maraging steel. The paper describes the effects of LSP on chosen $12 \% \mathrm{Ni}$ maraging steel, which is suitable also for the manufacture of tools for die casting of aluminium alloys. After laser treatment, measurements of residual stresses and microhardness and other properties, including surface defects at the micro level, were carried out. The results of the study confirmed that exceptionally favourable residual stresses and microhardness could be obtained.
\end{abstract}

Keywords: Laser Shock Processing; LSP; maraging steel; residual stresses; micro hardness.

Reference to this paper should be made as follows: Grum, J., Zupančič, M., Ocaña, J.L., Morales, M. and Porro, J.A. (2008) 'Laser shock processing as a method of decreasing fatigue of a die-casting die made of maraging steel', Int. J. Microstructure and Materials Properties, Vol. 3, Nos. 2/3, pp.271-281.

\section{Introduction}

To ensure an efficient Laser Shock Processing (LSP), the workpiece surface should be covered by an absorption layer, which should evaporate as fast and efficiently as possible under the action of the laser energy. This will produce a transformation of the laser-beam energy into a mechanical one. The evaporation of the absorption layer produces high pressure at the point of interaction if the power density is high enough. The absorption 
layer may be a dark-coloured coat of up to $0.1 \mathrm{~mm}$ in thickness. A transparent covering layer of water, glass or artificial resin makes it possible to limit a volume increase and through-depth compression directing. A usual thickness of the covering medium ranges between 2 and $4 \mathrm{~mm}$ or more. The laser beam applied to material shock processing shall be of a pulsed type and of high energy; the energy of an individual pulse usually ranges between some joules to some tens of joules, and exceptionally up to some 100 joules. The efficiency of transformation into the mechanical energy will increase with a high-power density and short pulse duration. This requires an accurate source and amplification of the laser beam. Typical pulse duration ranges between 0.6 and 30 nanoseconds, and power density is equal to more than $1 \times 10^{9}$ and over $100 \times 10^{9} \mathrm{~W} / \mathrm{cm}^{2}$. The process efficiency will be increased by short pulses of high-power density. The laser-beam diameter in practical applications usually ranges between 0.1 and $3.0 \mathrm{~mm}$ and more, and it is limited primarily by the laser-source power.

The effects of mechanical shock waves are similar to those of shot peening. The density of dislocations and, consequently, dynamic strength of the material will increase. LSP is essentially more expensive than shot peening, but it provides some advantages, e.g., the possibility of an accurate control of processing parameters and accomplishment of different transitions between the treated surface and the untreated one.

Shock-wave processing based on the pulsed laser has been discussed in several publications. Initially, publications discussed mainly the theoretical aspects, but the advancement of technical facilities has permitted studies on an increasingly large number of materials. Modelling of LSP was discussed, e.g., by Gámez and Ocaña (2000) and Fazal and Arif (2003), who described the key processing parameters using a model of computer simulation of the affected-layer depth and residual stresses in this layer.

Ocaña et al. (2004) discussed the influence of LSP on the increase of wear resistance of metals and metal alloys. The comprehensive research work also discussed plasma generation and a mode of a shock-wave propagation into the subsurface layers. Berthe et al. (2000) discussed the generation of plasma and shock waves from the viewpoint of physics under the circumstances in which wave propagation was directed and amplified by means of a covering layer of water. Also, Sollier $e$ t al. (2001) dealt with a physical description of a numerical plasma model in LSP. They particularly focused on the plasma changes in the final phase of laser-beam action, when the laser-beam energy transfer to the material surface is shadowed (»breakdown plasma«).

Montross et al. (2002) discussed residual internal stresses in a metal material after LSP. A theoretical approach to the occurrence of compressive residual stresses was supplemented with a discussion of the influence of individual parameters on the process, i.e., of laser-radiation wavelength $\lambda$, number of pulses, laser-spot size. Very interesting results were found in the wear of 7075-T7351 aluminium alloy after laser processing and classical processing. It was found that LSP provided an operating life that is several times longer.

\section{Experimental procedure}

\subsection{Preparation of specimens and measurements}

To determine the influence of LSP on final properties of maraging steel, several prismatic specimens were prepared. They were all produced in the same way and showed presumably the same microstructure, mechanical properties, and the variations of 
microhardness and residual stresses. The material chosen for this purpose was maraging steel with $12 \%$ of $\mathrm{Ni}$ and a Co-Mo-Ti system for precipitation hardening. The maraging steel was in as-delivered state which means that it was solution-annealed and homogenised by forging. The initial microstructure of samples was saturated nickel martensite with hardness of $30 \mathrm{HRC}$ to $32.5 \mathrm{HRC}$.

The specimens were cut out of a rod; the discs cut of $3.5 \mathrm{~mm}$ in thickness were ground and then cut with a grinder to their final size, i.e., $60 \times 30 \times 2.5 \mathrm{~mm}$. Both prior to and after LSP, the following measurements were carried out at each specimen:

- surface roughness (general assessment of surface quality, measurement of mean deviation of the profile $R_{a}$ )

- microhardness (HV2.0)

- through-depth profile of residual stresses (hole-drilling method)

- assessment of microstructure using optical and electronic microscopes.

LSP was performed using four different combinations of basic parameters. The combination of laser beam diameter and pulse densities is shown in Table 1. Measurements from within LSP-treated zone are additionally marked with 'ob' while measurements from non-treated zone are marked with ' $n$ '.

Table 1 Combinations of basic parameters for LSP

\begin{tabular}{lcc}
\hline Designation & Beam diameter $[\mathrm{mm}]$ & Pulse density $\left[\mathrm{pulses} / \mathrm{cm}^{2}\right]$ \\
\hline 1 & 1.5 & 1225 \\
2 & 1.5 & 5000 \\
3 & 2.0 & 1225 \\
4 & 2.0 & 5000 \\
\hline
\end{tabular}

After LSP, precipitation hardening was performed in a classical furnace at temperatures of $410^{\circ} \mathrm{C}, 450^{\circ} \mathrm{C}$ and $490^{\circ} \mathrm{C}$ for two hours. Precipitation annealing may drastically change the initial microstructure with precipitates that form within the nickel martensite. With the selected type of maraging steel different types of precipitated phases are present, such as $\mathrm{Fe}_{2} \mathrm{Mo}, \mathrm{Ni}_{3} \mathrm{Ti}, \mathrm{Ni}_{3} \mathrm{Mo}$, TiC, TiN and others. These precipitated phases significantly change mechanical and other properties, the most evident being higher strength and hardness. Microstructure is described in more detail by Grum and Zupančič (2002) with preceding tests and results explained for conventional precipitation hardening of the discussed maraging steel.

Finally, after precipitation annealing, a third set of measurements followed.

\subsection{Residual stresses after shock processing}

During processing a laser beam will first locally heat the absorption layer. An increase in temperature in the zone of interaction will produce local evaporation of the medium and its expansion. A high-power density and a short-term expansion will produce a shock wave to propagate into the covering layer as well as into the material below the absorption layer. The temperature in the zone of expansion in the time periods at the level of nanoseconds, which is typical of LSP, can be determined in accordance with Fourier's 
theory of heat transfer. With pulsed heating used in such a treatment, the temperature as a function of linear coordinate $\mathrm{x}$, time $\mathrm{t}$, material properties, and supply of heat flow by the laser-beam can be expressed as a non-linear differential equation of the second order, as explained by Montross et al. (2002). A fast temperature increase will result in rapid expansion at the point of interaction of the laser beam with the surface, the $\mathrm{V}$ being the velocity of moving away of the surface, which is a result of expansion and can be determined from the energy equilibrium in the zone of interaction, and $\mathrm{L}_{\mathrm{ev}}$ is the latent heat consumed in the evaporation of the absorption layer. Considering the mode of calculation, boundary conditions and the limitations taken into account in modelling, the calculation of basic quantities may be very complex. A complete analytical solution of such an equation for non-uniform boundary conditions of evaporation is not possible; a partial solution, however, can be applied using the Laplace transformation of the equation of temperature in time.

The through-depth propagation of the mechanical shock wave in the material produces local plastic deformation at the micro level that, in turn, produces a local change of the dislocation density. The plastic deformation will occur to the depth where the shock wave can still cross the so-called Hugoniot's Elastic Limit (HEL), also dealt with by Johnson and Rhode (1971). The magnitude of the stress owing to the compressive shock-wave is conditioned exclusively by the material properties, i.e., the dynamic yield strength and Poisson's ratio. A higher Poisson's ratio will require a higher shock-wave compression. As maraging steel has a similar Poisson's ratio as common steel, this means that the shock-wave compression should be approximately $70 \%$ higher than the dynamic yield strength to achieve plastic deformation.

The stresses in the surface layer can be determined according to Ballard et al. (1991). The magnitude of the residual stresses in the thin surface layer owing to shock waves depends on the material properties (Lamé constants, the velocity of elastic and plastic deformation) and on the process conditions (time of compressive-wave interaction, beam radius at the surface, shock-wave compression). Changes in the processing parameters that increase the stresses in the surface layer are primarily the lengthening of the time of interaction, increases in beam diameter and shock-wave compression. The fulfilment of these requirements is in opposition to the requirements for the achievement of a greater affected depth: thus for example, a larger beam radius will require a shorter time of interaction if the same energy density is to be kept.

In practice, the residual stresses in the surface layer owing to LSP can differ considerably and can range between 0 and the yield stress of the material concerned. The equations for calculations are considered valid under the condition that $\mathrm{p}_{\mathrm{uv}}>2 \mathrm{HEL}$. When this condition is fulfilled, the compression will certainly be strong enough to produce the effects desired.

The stresses after grinding, i.e., prior to LSP, were primarily tensile residual stresses of relatively low values. In almost all cases they were limited to below $100 \mathrm{MPa}$ of the absolute value in the surface layer. This stress, however, was reduced with depth. The measurements of the angles of the principal stresses also showed that in all the cases, the angles were related to the direction of treatment, i.e., with the parameters chosen, the orientation of all the maximum stresses was perpendicular to the direction of the grinder movement. 
Figure 1 shows the LSP conditions, the measured residual-stress profiles and a through-depth stress gradient, the laser beam used having a diameter of $1.5 \mathrm{~mm}$. The residual-stress profile after LSP was changed considerably. The stress profiles shifted to the compressive zone to depths exceeding $0.2 \mathrm{~mm}$, a smaller influence being seen also in a depth exceeding $1 \mathrm{~mm}$ (Figure 1A). Figure 1(B) shows the differences among residual stresses in the $0.15 \mathrm{~mm}$ thick surface layer and those in the chosen reference depth of $0.5 \mathrm{~mm}$; an essential increase in the through-depth stress gradient may be observed. An increase in compressive residual stresses is desired, but the through-depth stress gradient should not be too high because it may produce microcracks and, in exceptional cases, also peeling of the surface layer.

Figure 1 (A) Residual-stress profiles after LSP. (B) Differences among stresses in $0.15 \mathrm{~mm}$ thick surface layer and those in depth of $0.5 \mathrm{~mm}$ after LSP with $1.5 \mathrm{~mm}$ beam

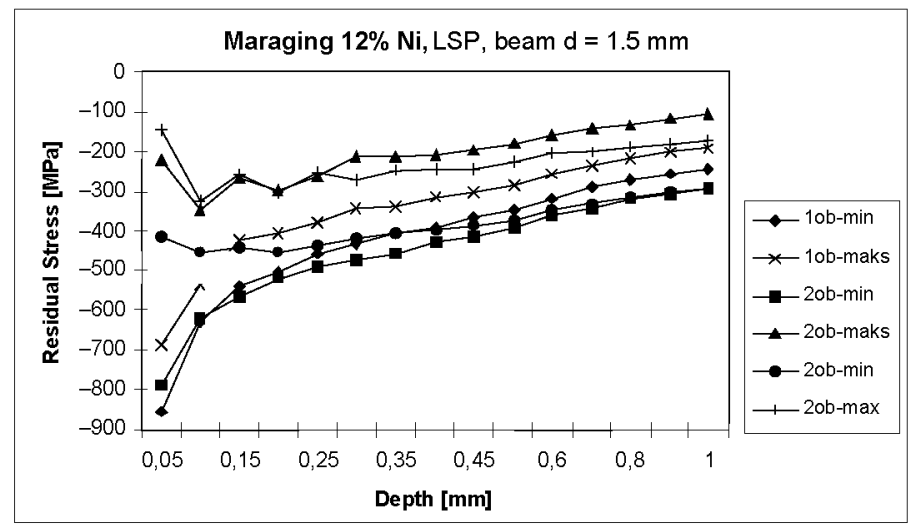

(A)

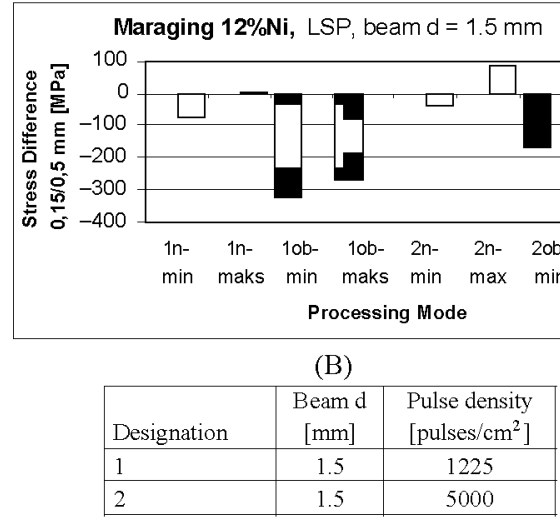

Note: Measurements from within LSP-treated zone are marked with 'ob' while measurements from non-treated zone are marked with ' $n$ '.

The angle of the principal residual stresses can be an indicator of the changes occurring during the treatment in the surface layer. The angles of the principal stresses are, similarly to stress profiles after LSP, essentially changed with reference to the initial state. The depths where stronger changes of the angle occur are those where during LSP, changes of the stress state have occurred. 
Figure 2(A) shows the residual-stress profiles and the gradient of the residual stresses in the surface layer after LSP, the laser-beam diameter being $2.0 \mathrm{~mm}$. The residual-stress profile is similar to the first case, which means that no essential difference caused by the change of beam diameter could be noticed. But there is a great difference owing to the number of pulses, i.e., density, during the treatment with $1225 \mathrm{pulses} / \mathrm{cm}^{2}$ and that of $5000 \mathrm{pulses} / \mathrm{cm}^{2}$. The residual stresses under Conditions 3 , where the pulse density used was 1225 pulses $/ \mathrm{cm}^{2}$, are absolutely smaller than those under Conditions 4 , where the pulse density used was 5000 pulses $/ \mathrm{cm}^{2}$. The gradients of the residual stresses in different depths (Figure 2B) are considerably greater than before LSP also in the case of the $2.0 \mathrm{~mm}$ laser beam. From a comparison of the data on the residual stresses, it can be inferred that the influence of the laser-beam size is comparatively small whereas the influence of pulse density on the residual stresses is very strong.

Figure 2 (A) Residual-stress profiles after LSP. (B) Differences among stresses in $0.15 \mathrm{~mm}$ thick surface layer and those in depth of $0.5 \mathrm{~mm}$ after LSP with $2.0 \mathrm{~mm}$ beam

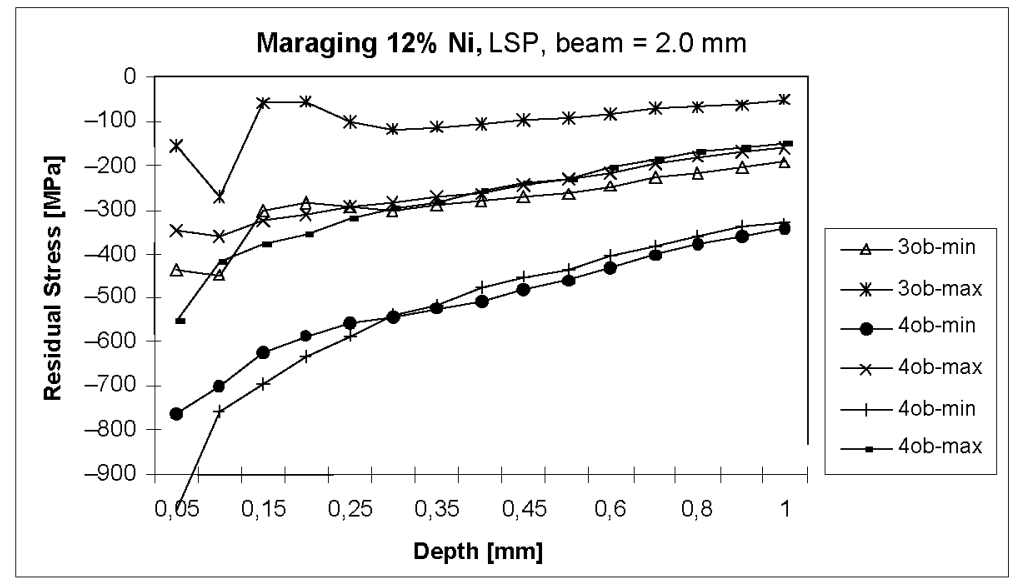

(A)

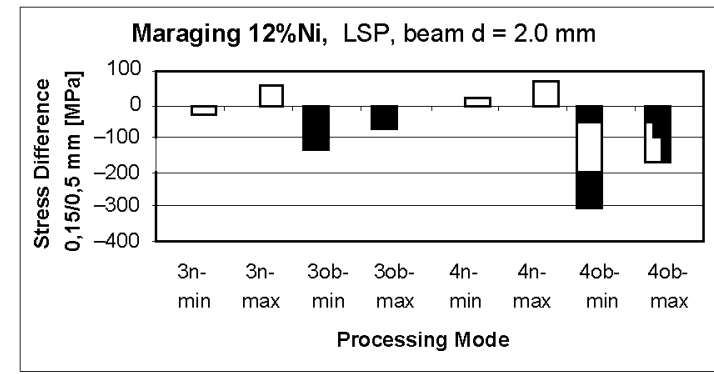

(B)

\begin{tabular}{|l|c|c|}
\hline Designation & $\begin{array}{c}\text { Beam d } \\
{[\mathrm{mm}]}\end{array}$ & $\begin{array}{c}\text { Pulse density } \\
{\left[\text { pulses } / \mathrm{cm}^{2}\right]}\end{array}$ \\
\hline 3 & 2.0 & 1225 \\
\hline 4 & 2.0 & 5000 \\
\hline
\end{tabular}

Note: Measurements from within LSP-treated zone are marked with 'ob' while measurements from non-treated zone are marked with ' $n$ '. 
The residual stresses in the surface layer, if compared to the stress in the depths of $0.50 \mathrm{~mm}$ and $1.00 \mathrm{~mm}$ respectively, are considerably higher after LSP than before processing. The differences are smaller if the surface layer of a greater thickness $(0.15 \mathrm{~mm})$ is compared to a smaller reference depth $(0.50 \mathrm{~mm})$; on the contrary, they are greater if the thin surface layer $(0.10 \mathrm{~mm})$ is compared to a greater reference depth $(1.00 \mathrm{~mm})$. High compressive stresses in the surface layer are otherwise desired, but in this case they also show a high through-depth gradient of stresses, which is not quite favourable.

The conditions in the surface layer after LSP and additional precipitation annealing are shown in Figure 3. The profiles of the principal residual stresses are lying in a completely compressive zone and can be, in the surface layer, close to the yield stress of the material chosen. As anticipated, a greater number of the pulses employed in LSP increases the depth affected. Figure 3(A) shows the profiles measured of the minimum residual stresses after LSP with the $1.5 \mathrm{~mm}$ beam and the number of pulses being $1225 \mathrm{~cm}^{-2}$ and $5000 \mathrm{~cm}^{-2}$ and additional annealing at $410^{\circ} \mathrm{C}$ for two hours. A greater number of pulses produces a considerable increase of the depth affected and, consequently, a similar or even lower stress gradient in the surface layer. With the specimens treated with the $2.0 \mathrm{~mm}$ beam after precipitation annealing, the affected depth is much greater with a higher pulse density, and the stress gradient in the surface also increases a little, as shown in Figure 3(B). Thus it can be confirmed that a greater number of pulses during LSP with given processing parameters and material employed does not necessarily increase the magnitude of the residual stresses in the surface layer, but it has a favourable influence on the residual-stress profile and increases the depth affected.

Figure 3 Residual-stress profiles after LSP and additional precipitation annealing in furnace at $410^{\circ} \mathrm{C} / 2 \mathrm{~h}$

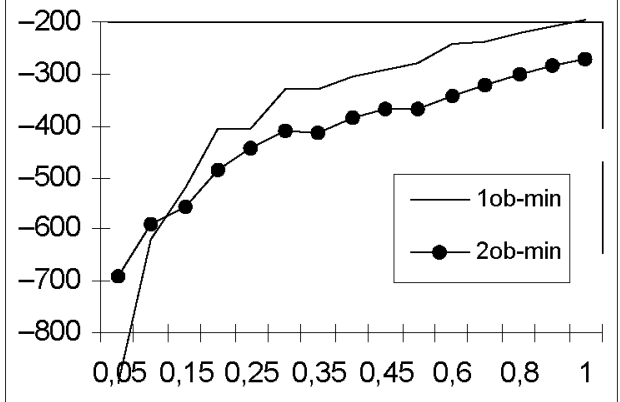

(A) Minimum stresses after LSP with $1.5 \mathrm{~mm}$ beam and $1225 \mathrm{pulses} / \mathrm{cm}^{2}$ (processing parameters 1 ) and $5000 \mathrm{pulses} / \mathrm{cm}^{2}(2)$

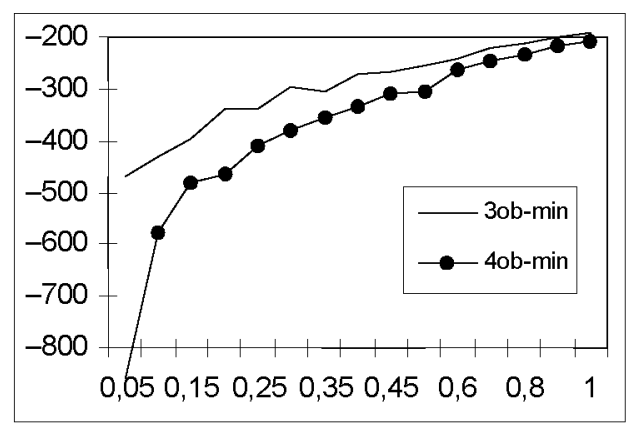

(B) $2.0 \mathrm{~mm}$ beam and 1225 pulses/ $\mathrm{cm}^{2}$ (parameters 3 ) and $5000 \mathrm{pulses} / \mathrm{cm}^{2}$ (4)

\subsection{Microhardness after LSP and precipitation annealing}

Measurements of microhardness at the specimens after LSP indicate a change of the phases precipitated to a depth of approximately $0.5 \mathrm{~mm}$, which is in agreement with the measurements of the residual stresses. Figure 4(A) shows the microhardness variation in the thin surface layer after LSP. It shows the conditions prior to and after additional 
precipitation annealing in the furnace. Increasing of microhardness in the surface layer can be observed to a depth of approximately $0.5 \mathrm{~mm}$ (Figure 4A). This increase in microhardness in the surface layer after LSP is true for processing conditions with LSP (Conditions 1, 2, 3 and 4). After the additional precipitation annealing, hardness will increase both in the affected surface layer and in a greater depth. The change of microhardness is different with different modes of processing. If measurement deviations that may occur because of a small load in microhardness measurement are excluded, this may be explained by the influence on the formation of the phases precipitated, which may, owing to the effects of shock waves, precipitate at several locations with an increased density of dislocations. This results in a greater density of the precipitated phases, which are on the average smaller and increase microhardness to a smaller degree than in the greater depth.

Figure 4 Microhardness (HV2.0) after LSP (A) and after additional precipitation annealing at $410^{\circ} \mathrm{C}(\mathrm{B}), 450^{\circ} \mathrm{C}(\mathrm{C})$ and $490^{\circ} \mathrm{C}$ (D) for two hours

Microhardness after LSP

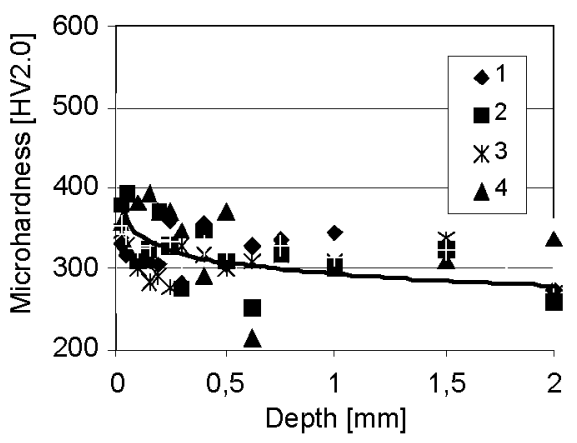

(A)
LSP $+410^{\circ} \mathrm{C} / 2 \mathrm{~h}$

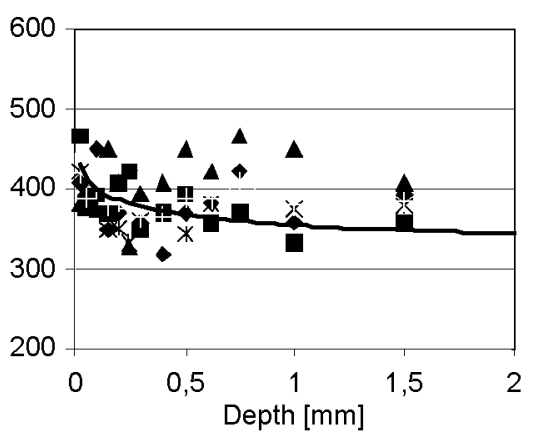

(B)
$\mathrm{LSP}+450^{\circ} \mathrm{C} / 2 \mathrm{~h}$

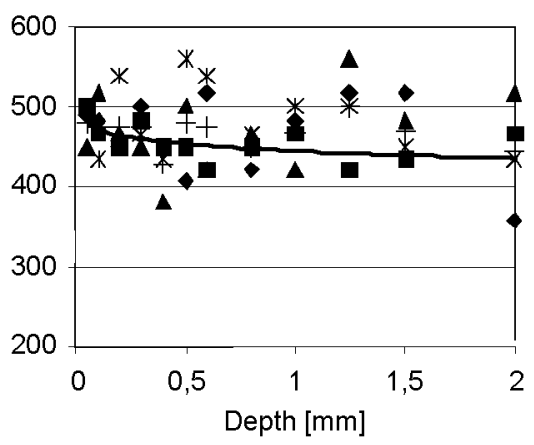

(C)
$\mathrm{LSP}+490^{\circ} \mathrm{C} / 2 \mathrm{~h}$

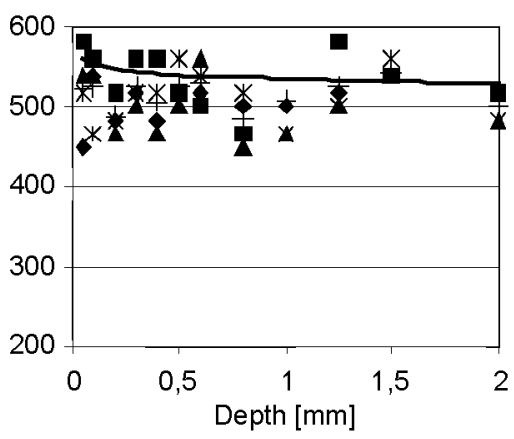

(D)

Note: For clarity of the results, only one trendline is drawn (for Conditions 2: beam diameter $1.5 \mathrm{~mm}$ and pulse density $5000 \mathrm{~cm}^{-2}$ ). 
The individual microhardness diagrams show stronger changes between the hardness in the depth of $0.5 \mathrm{~mm}$ and that in a depth exceeding $1 \mathrm{~mm}$; it can be observed that shock waves produce a change of microhardness also after precipitation annealing which is, however, smaller with higher temperature of precipitation annealing.

The difference between the results obtained for the microhardness in the affected surface layer and those obtained in the core was statistically evaluated. A t-test was applied to a various number of microhardness measurements at the individual specimens in the surface layer and in the layer in the greater depth (exceeding $0.5 \mathrm{~mm}$ ). A normal distribution of microhardness results and a mutual independence of the results of the two groups compared were presupposed. The statistical tests of the differences between the surface layer and the core showed that there was a significant difference in microhardness between the affected layer and the core both before and after precipitation annealing. For the hardness found immediately after LSP and after precipitation annealing at $410^{\circ} \mathrm{C}$ and $450^{\circ} \mathrm{C}$ significant differences were confirmed with a probability of error of 0.05 . After precipitation annealing at $490^{\circ} \mathrm{C}$, the probability that the difference is not significant equals 0.15 .

\section{Conclusions}

LSP has turned out to be a very promising way of treatment in order to improve surface properties of structural and tool materials. Shock waves produce locally oriented mechanical waving, propagating through the material depth. This results in changes of the material microstructure, including through-depth changes of dislocation density owing to the effects of shock waves.

The purpose of the tests performed on $12 \%$ maraging steel was to evaluate some of the hardening effects of LSP on the thin surface layer of the chosen maraging steel. The residual stresses after LSP are shifted to the compressive zone. High values of the residual stresses after LSP were present down to the depth of about $0.25 \mathrm{~mm}$, but changes were visible also in the depth over $0.50 \mathrm{~mm}$. The pulse density increases the absolute values of residual stresses and makes sure that the transition from the compressive zone to the tensile one will occur in a greater depth. Consequently, owing to the greater affected depth, even lower gradients of residual stresses in the thin surface layer may be obtained.

After LSP, subsequent precipitation hardening was performed. Precipitations at relatively low temperature of $410^{\circ} \mathrm{C}$ for two hours produce higher hardness for about 100 HV2.0 regarding the initial state. After such hardening, residual stress profiles change only slightly, which could be very interesting; this means that it is possible to perform surface laser peening of the maraging steel in a soft state and to subsequently harden it also with precipitates. This way, an increase in the fatigue strength under thermo-mechanical loads can be expected. Precipitation temperatures above $450^{\circ} \mathrm{C}$ produce higher hardness, but unfortunately also made the effect of stress relaxation more pronounced. This is especially valid for even higher temperature of $490^{\circ} \mathrm{C}$ or more. 


\section{References}

Ballard, P., Fournier, J., Fabbro, R. and Frelat, J. (1991) 'Residual stresses induced by laser shocks', Journal de Physique, Vol. IV, No. 1, pp.487-494.

Berthe, L., Sollier, A., Peyre, P., Fabbro, R. and Bartnicki, E. (2000) 'The generation of laser shock waves in a water-confinement regime with 50 ns and 150 ns $\mathrm{XeCl}$ excimer laser pulses', J. Phys. D: Appl. Phys., Vol. 33, pp.2142-2145.

Fazal, A. and Arif, M. (2003) 'Numerical prediction of plastic deformation and residual stresses induced by laser shock processing', Journal of Materials Processing Techn., Vol. 136, pp.120-138.

Gámez, B. and Ocaña, J.L. (2000) 'A theoretical method for the calculation of frequency- and temperature-dependent interaction constants applicable to the predictive assessment of laser materials processing', J. Phys. D: Applied Physics, Vol. 33, pp.305-312.

Grum, J. and Zupančič, M. (2002) 'Suitability assessment of replacement of conventional hot-working steels with maraging steel. Part 2, microstructure of maraging steel after precipitation hardening treatment', Z. Met.kd., Vol. 93, No. 2, pp.171-176.

Johnson, J.N. and Rhode, R.W. (1971) 'Dynamic deformation twinning in shock loaded iron', Journal of Applied Physics, Vol. 42, pp.4171-4182.

Montross, C.S., et al. (2002) 'Laser shock processing and its effects on microstructure and properties of metal alloys: a review', International Journal of Fatigue, Vol. 24, pp.1021-1036.

Ocaña, J.L., Morales, M., Molpeceres, C., Torres, J., Porro, J.A., Gomez, G. and Rubio, C. (2004) 'Predictive assessment and experimental characterization of the influence of irradiation parameters on surface deformation and residual stresses in laser shock processed metallic alloys', Proceedings of the SPIE, Vol. 5448, pp.642-653.

Sollier, A., Berthe, L. and Fabbro, R. (2001) "Numerical modeling of the transmission of breakdown plasma generated in water during laser shock processing', Eur. Phys. J. AP, Vol. 16, pp.131-139. 